\title{
Expedient Synthesis of 5-Benzoylpyrimidine-2,4-diones from Baylis-Hillman Adducts
}

\author{
Jeong Mi Kim, Eun Sun Kim, and Jae Nyoung Kim* \\ Department of Chemistry and Institute of Basic Science, Chonnam National Chiversity, Gwangiu 500-757, Korea \\ E-mail: kiminachonnam ack $k r$ \\ Recened November 7, 2008, Accepted Februarv 6, 2009
}

Key Words: Baylis-Hillman adducts. Pyrimidine-2,4-diones, PCC oxidation

The sỵthesis and modification of pyrrimidine-2.4-dione (uracil) derivatives has received much attention due to their importance in nucleic acid chemistry as well as in synthetic organic chemistry. ${ }^{1-3}$ Most of the modifications involved the introduction of various substituents at the 5- or 6-position of uracil ring. ${ }^{1 . \hat{}}$ Recently numerous chemical transformations of Baylis-Hillman adducts have been published involving the synthesis of various heterocyclic compounds.

The synthesis of 5-benzyluracil 2a starting from BaylisHillman adduct was reported by us recently. 5-Benzoyluracil and related compounds are also important. ${ }^{a, b, 2}$ thus we examined the oxidation of 5-benzyluracil 2a into 5-benzoyl derivative $4 \mathbf{a}$ as in Scheme 1. The reaction of 2a with $\mathrm{SeO}_{2}$ or $\mathrm{KMnO}_{4}$ showed no reaction while with $\mathrm{PCC}$ (pyridinium chlorochromate) or $\mathrm{CrO}_{3} / \mathrm{AcOH}$ produced low yield of product. $^{6}$

Thus we decided to prepare $\mathbf{4 a}$ by the oxidation of 5-benzylideneuracil derivative 3 a with $\mathrm{PCC}$. which was efficiently used for the oxidation of similar compounds by us recently. As reported. 5-benzyl derivative 2a was obtained from la with strong base such as NaOEt or $t$-BuOK. ${ }^{5}$ When the reaction of 1a was carried out under the influence of $\mathrm{K}_{2} \mathrm{CO}_{3}$ in $\mathrm{DMF}$ at elevated temperature, 5-benzyl derivative 2a was the major product again. Fortunately. 5-benzylidene compound 3 a was obtained as the major product $(82 \%)$ when we nun the reaction under the influence of a catalytic amount of $\mathrm{K}_{2} \mathrm{CO}_{3}(0.2$ equiv) in DMF at room temperature. With this benzylidene compound 3a. we examined the PCC oxidation and obtained the benzoyl derivative ta in good yield (78\%). Encouraged by the results we prepared $3 \mathrm{~b}-\mathrm{f}$ and examined the oxidation to 5 -benzoyluracils $\mathbf{4 b - f}$ and the results are summarized in Scheme 2 and Table 1.

The synthesis of starting materials $\mathbf{1 b} \mathbf{b}$ was carried out as reported. ${ }^{5}$ Cyclization of $\mathbf{1 b}$-e was carried out under the same conditions $\left(\mathrm{K}_{2} \mathrm{CO}_{3}\right.$. DMF, rt. $\left.8 \mathrm{~h}\right)$, and we obtained $3 \mathrm{~b}-\mathrm{e}$ in $63-92 \%$ yields. However. 5-benzyl derivative was formed as the major product when we run the reaction of thiourea derivative if under the same conditions even at room temperature presumably by the base-mediated isomerization process of $3 \mathrm{f}$. Thus we carried out the reaction in water without base at refluxing tentperature for long time for the synthesis of $\mathbf{3 f}$. With these benzylidene compounds $\mathbf{3 b} \mathbf{b}$, the following oxidation was carried out with PCC (2.0 equiv) in $\mathrm{CH}_{2} \mathrm{Cl}_{2}$ at refluxing temperature to obtain $4 \mathrm{~b}-\mathrm{g}$ in $63-80 \%$ yields. As in entries $1-4.1$-substituents $\left(\mathrm{R}_{1}\right.$ and $\left.\mathrm{R}_{2}\right)$ did not affect the reactivity and the reactions with hexylidene

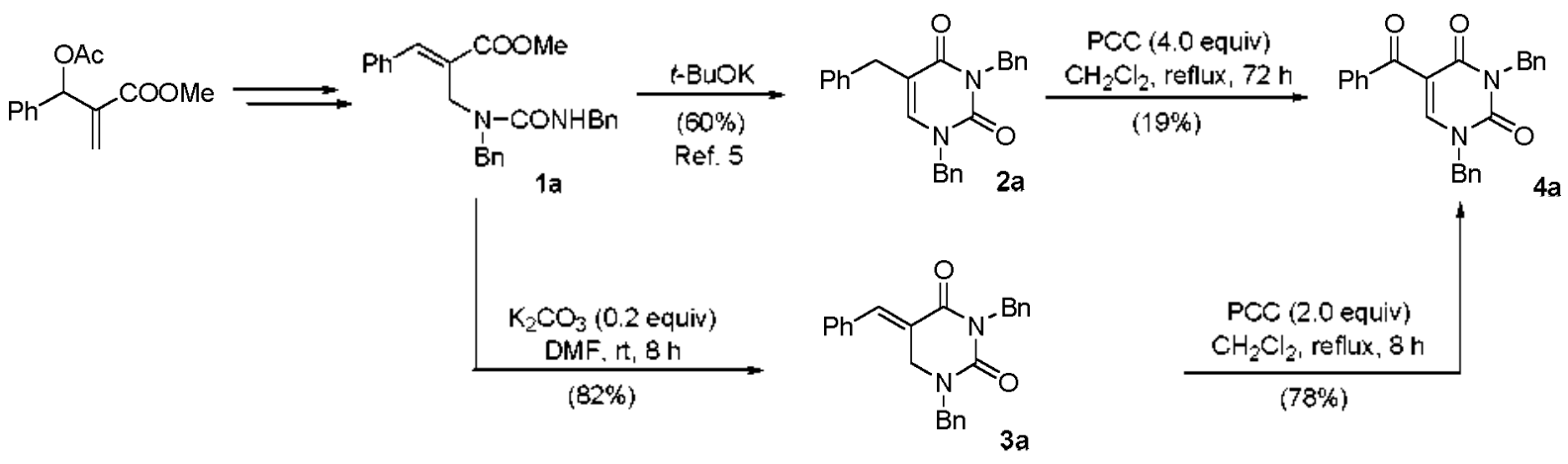

Scheme 1

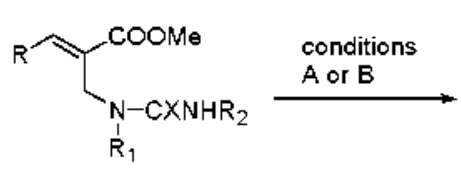

$1 a-f$<smiles>[R]C=C1CN([R1])C([X])N([R])C1=O</smiles>

$4 a-f$

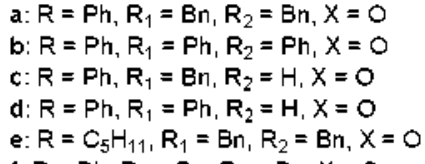

Scheme 2 
Table 1. Synthesis of 5-benzylidene- and 5-benzoyl pvrimidines

\begin{tabular}{ccccc}
\hline Entry & Substrate 1 & Conditions $^{\sigma}$ & $\begin{array}{c}\text { Compound 3 } \\
(\%)\end{array}$ & $\begin{array}{c}\text { Product + } \\
(\%)^{2}\end{array}$ \\
\hline 1 & 1a & A & $\mathbf{3 a}(82)$ & $\mathbf{4 a}(78)$ \\
2 & 1b & A & $\mathbf{3 b}(92)$ & $\mathbf{4 b}(73)$ \\
3 & $1 \mathrm{c}$ & $\mathrm{A}$ & $\mathbf{3 c}(90)$ & $\mathbf{4}(63)$ \\
4 & 1d & A & $\mathbf{3 d}(90)$ & $\mathbf{4 d}(80)$ \\
5 & 1e & A & $\mathbf{3 e}(63)$ & $\mathbf{4 e}(65)$ \\
6 & 1f & B & $\mathbf{3 f}(64)$ & $\mathbf{4 f}(66)$ \\
\hline
\end{tabular}

${ }^{a}$ Conditions $\mathrm{A}: \mathrm{K}_{2} \mathrm{CO}_{3}$ (0.2 equit), DMF, rt. 8 h: Conditions $\mathrm{B}: \mathrm{H}_{2} \mathrm{O}$. reflux. 48 h. ${ }^{\circ}$ Conditions: $\mathrm{PCC}$ (2.0 equiv), $\mathrm{CH}_{2} \mathrm{Cl}_{2}$, reflux, $8 \mathrm{~h}$.

derivative (entry 5) and thiourea derivative (entry 6) also showed same reactivity.

In summary, we developed an efficient way for the preparation of 5-benzoylpy rimidine-2 4-dione derivatives from BaylisHillman adducts by using PCC oxidation of 5-benzylidene derivatives as the key step.

\section{Experimental Section}

Sy nthesis of starting materials was carried out as reported ${ }^{5}$ and the spectroscopic data of unknown compounds. 1b. 1e and 1f are as follows. Compounds 1a-d and 1f were obtained as pure $E$ isomers, but compound le was separated as an $E Z$ mixture ( $E: Z=4: 1$ ). However. compound $3 \mathrm{e}$ ( $E$ form) could be isolated in pure state from the corresponding $Z$-3e derived from the minor $Z$-1e

Compound 1b: $80 \%$; colorless oil: IR (film) 3424. 1716. $1681 \mathrm{~cm}^{-1}$ : ${ }^{1} \mathrm{H} N \mathrm{NM}\left(\mathrm{CDCl}_{3}, 300 \mathrm{MHz}\right) 83.73(\mathrm{~s}, 3 \mathrm{H}), 4.98(\mathrm{~s}$, 2H). 6.35 (s. IH). 6.95-7.07 (m. 3H). 7.13-7.37 (m. 12H). $7.71(\mathrm{~s}, 1 \mathrm{H}):{ }^{13} \mathrm{C}$ NMR $\left(\mathrm{CDCl}_{3} .75 \mathrm{MHz}\right)$ ò $44.12,52.14$. $119.28,122.84 .128 .26$ (2C). 128.41, 128.75, 128.87, 129.13. $129.26,129.82,134.41,138.87,139.75,143.43,154.22$. 168.16

Compound 1e: $94 \%$ : colorless oil: IR (film) 3358. 1716. $1645 \mathrm{~cm}^{\cdot .}$ : ${ }^{1} \mathrm{H}$ NMR (major $E, \mathrm{CDCl}_{3} .300 \mathrm{MHz}$ ) $\delta 0.86$ (t. $J=$ $6.9 \mathrm{~Hz} .3 \mathrm{H}) .1 .15-1.43(\mathrm{~m}, 6 \mathrm{H}) .2 .12(\mathrm{q} . J=7.5 \mathrm{~Hz}, 2 \mathrm{H}), 3.67$ $(\mathrm{s}, 3 \mathrm{H}), 4.11(\mathrm{~s}, 2 \mathrm{H}), 4.42-4.50(\mathrm{~m} .+\mathrm{H}), 6.20(\mathrm{t} . J=5.1 \mathrm{~Hz}$. IH). $6.90(\mathrm{t}, J=7.5 \mathrm{~Hz}, \mathrm{IH}) .7 .15-7.33(\mathrm{~m}, 10 \mathrm{H}):{ }^{1} \mathrm{H}$ NMR (minor Z. $\mathrm{CDCl}_{3} .300 \mathrm{MHz}$ ) $\delta 0.89$ (t, $J=6.9 \mathrm{~Hz} .3 \mathrm{H}$ ). $1.15-1.43(\mathrm{~m}, 6 \mathrm{H}), 2.43(\mathrm{q} . J=7.2 \mathrm{~Hz}, 2 \mathrm{H}), 3.66(\mathrm{~s}, 3 \mathrm{H}), 4.00$ (s. 2H). $4.42-4.50(\mathrm{~m}, 4 \mathrm{H}) .5 .47$ (t. $J=5.4 \mathrm{~Hz}, \mathrm{IH}) .6 .01$ (t. $J$ $=7.5 \mathrm{~Hz}, 1 \mathrm{H}) \cdot 7.15-7.33(\mathrm{~m} .10 \mathrm{H}):{ }^{13} \mathrm{C} \mathrm{NMR}$ (major + minor, $\mathrm{CDCl}_{3 .}, 75 \mathrm{MHz}$ ) ò $13.76,13.84,22.22,22.25,28.34,28.49$. $28.66,29.33 .31 .24,31.34 .42 .15 .44 .81,44.91 .48 .78,49.26$, $49.56,51.36,51.90,126.49,126.75,126.89 .126 .92,127.07$. $127.34,127.39,127.52,127.77,128.24,128.31,128.37$. $128.44 .137 .87,138.00,139.57 .139 .71,145.68,147.66$. $158.36,158.99,167.51,168.36$ ( $\mathrm{lC}$ is overlapped).

Compound 1f: $96 \%$ : colorless oil; $\mathbb{R}$ (film) $3411,3304$. 1713. 1697, 1257, $1202 \mathrm{~cm}^{-1}:{ }^{1} \mathrm{H} \mathrm{NMR}\left(\mathrm{CDCl}_{3}, 300 \mathrm{MHz}\right) \dot{o}$ 3.68 (s. $3 \mathrm{H}$ ). 4.50 (s. $2 \mathrm{H}$ ), 4.83 (d. $J=4.8 \mathrm{~Hz} .2 \mathrm{H}$ ). 4.90 (s. 2H). 6.89-6.92 (m. 2H), 6.99-7.01 (m, 2H), 7.04-7.11 (m. $3 \mathrm{H}) .7 .17-7.33(\mathrm{~m}, 9 \mathrm{H}) .7 .8 \mathrm{l}(\mathrm{s}, 1 \mathrm{H}):{ }^{13} \mathrm{C} \mathrm{NMR}\left(\mathrm{CDCl}_{3 .} 75\right.$ $\mathrm{MHz})$ ò 43.95. 50.09, 52.14. 53.51. 126.51, 126.81, 126.85 . $127.29,127.42,127.77,128.08,128.31,128.58,128.85$. $133.14 .136 .11 .137 .84 .143 .75,167.96,183.95$.
Typical procedure for the synthesis of $3 \mathrm{a}$. A nuxture of 1 a (207 $\mathrm{mg} .0 .5 \mathrm{mmol}$ ) and $\mathrm{K}_{2} \mathrm{CO}_{3}(14 \mathrm{mg} .0 .1 \mathrm{mmol}$ ) in DMF $(3.0 \mathrm{~mL})$ was stirred at room temperature for $8 \mathrm{~h}$. After the usual aqueous workup and colunn chromatographic purification process (hexanes/ether. $1: 1$ ) compound 3a was obtained as a white solid. $157 \mathrm{mg}$ ( $82 \%$ ). Other compounds were synthesized similarly and their spectroscopic data are as follows.

Conpound 3a: $82 \%$, white solid. mp 122-124 ${ }^{\circ} \mathrm{C}$ : $\mathrm{RR}(\mathrm{KBr})$ 1699. 1667, $1216 \mathrm{~cm}^{.]}$: ' H NMR (CDCl $\left.3.300 \mathrm{MHz}\right) \delta 4.23(\mathrm{~d}$, $J=2.4 \mathrm{~Hz} .2 \mathrm{H}$ ). 4.65 (s. $2 \mathrm{H}), 5.15$ (s. $2 \mathrm{H}) .7 .14-7.17(\mathrm{~m}, 2 \mathrm{H}$ ). $7.23-7.40(\mathrm{~m}, 11 \mathrm{H}), 7.46-7.50(\mathrm{~m}, 2 \mathrm{H}) .7 .84(\mathrm{t}, J=2.4 \mathrm{~Hz}$, $\mathrm{lH}):{ }^{13} \mathrm{C} \mathrm{NMR}\left(\mathrm{CDCl}_{\underline{z}}, 75 \mathrm{MHz}\right) \hat{\delta} 44.62,44.63,51.65$. 122.82. 127.28. 127.84, 128.01, 128.36. 128.73, 128.74, 128.82, 129.42. 129.70, 134.03, 135.94. 137.88, 138.80. 152.71. 163.91: ESIMS $m / z 383\left(\mathrm{M}^{+}+\mathrm{H}\right)$.

Compound 3b: $92 \%$; white solid mp $198-200^{\circ} \mathrm{C}$; IR (KBr) $1713.1678,1225 \mathrm{~cm}^{-1}:{ }^{1} \mathrm{H}$ NMR (CDCl $\left.\mathrm{z}_{+} 300 \mathrm{MHz}\right) \hat{\delta} 4.87$ (d. $J=2.1 \mathrm{~Hz} .2 \mathrm{H}), 7.21-7.49($ m. $15 \mathrm{H}), 7.99(\mathrm{t}, J=2.1 \mathrm{~Hz}, 1 \mathrm{H})$; ${ }^{13} \mathrm{C} \mathrm{NMR}\left(\mathrm{CDCl}_{2} .75 \mathrm{MHz}\right) \hat{\delta}+7.96,122.95,125.62 .126 .93$, 128.28. 128.82(2C), 128.90. 129.09. 129.69, 129.85, 133.78 . $135.75 .139 .7+1+1.42 .151 .99,164.20$

Compound 3c: $90 \%$ : white solid. mp $185-187^{\circ} \mathrm{C}$ : $\mathbb{R}(\mathrm{KBr})$ $3200.1699,1261 \mathrm{~cm}^{\cdot 1}$ : ${ }^{1} \mathrm{H}$ NMR $\left(\mathrm{CDCl}_{3} .300 \mathrm{MHz}\right) \delta 4.29(\mathrm{~d}$, $J=2.1 \mathrm{~Hz}, 2 \mathrm{H}), 4.65$ (s. $2 \mathrm{H}), 7.18-7.43(\mathrm{~m} .10 \mathrm{H}) .7 .86$ (t. $J=$ $2.1 \mathrm{~Hz}, 1 \mathrm{H}) .8 .0+(\mathrm{s}, \mathrm{lH}) ;{ }^{13} \mathrm{C} \mathrm{NMR}\left(\mathrm{CDCl}_{3} .75 \mathrm{MHz}\right) \hat{\mathrm{o}}+5.52$. $50.57,122.05 .127 .96 .128 .08,128.83,128.89,129.73,129.92$. $133.78,135.70,139.24,151.69,163.98$.

Compound 3d: $90 \%$ : white solid, $\operatorname{mp} 295-297^{\circ} \mathrm{C}$ (dec.); IR (KBr) $3149.1682,1373 \mathrm{~cm}^{-1}:{ }^{1} \mathrm{H}$ NMR (DMSO-d $300 \mathrm{MHz}$ ) $\delta 4.80(\mathrm{~d}, J=7.2 \mathrm{~Hz} .2 \mathrm{H}) .7 .24-7.31(\mathrm{~m}, 1 \mathrm{H}) .7 .37-7.47(\mathrm{~m}$. 9H). 7.74 (t. $J=7.2 \mathrm{~Hz}, \mathrm{IH}) .10 .70$ (s. $1 \mathrm{H}$ ): ${ }^{13} \mathrm{C} \mathrm{NMR}$ (DMSO-d $6.75 \mathrm{MHz}$ ) ô 48.59, 123.68. 125.96. 126.46, 128.84, 128.89. 129.58. 130.17, 133.75, 136.64. 141.58, 151.14, 164.01: ESIMS $m / 2279\left(\mathrm{M}^{-}+\mathrm{H}\right)$.

Compound 3e: $63 \%$; colorless oil; IR (film) 1704, 1666 , $1453 \mathrm{~cm}^{-1}:{ }^{1} \mathrm{H} \mathrm{NMR}\left(\mathrm{CDCl}_{3}, 300 \mathrm{MHz}\right) \delta 0.85(\mathrm{t} . J=7.2 \mathrm{~Hz}$. $3 \mathrm{H}) .1 .15-1.43(\mathrm{~m}, 6 \mathrm{H}), 1.96-2.03(\mathrm{~m}, 2 \mathrm{H}), 3.88-3.90(\mathrm{~m}$, $2 \mathrm{H}$ ). 4.66 (s. 2H) 5.09 (s. 2H). $6.90-6.97(\mathrm{~m}, \mathrm{lH}) .7 .21-7.37$ (m. 8H), 7.42-7.46 (m, 2H): ${ }^{3} \mathrm{C}$ NMR $\left(\mathrm{CDCl}_{2}, 75 \mathrm{MHz}\right) \delta$ $13.85,22.31,27.76 .28 .01,31.31,43.10 .44 .34,51.43$, $122.70,127.17,127.79,127.92,128.29,128.66,128.78$. $136.06 .137 .98 .142 .91 .153 .05,163.39$

Compound 3f: $64 \%$ : white solid mp 136-138 $\mathrm{C}$ : IR (KBr) 1686. $1+48,1174 \mathrm{~cm}^{-1}$ : ${ }^{1} \mathrm{H}$ NMR $\left(\mathrm{CDCl}_{2}, 300 \mathrm{MHz}\right) \hat{o}+37$ (d, $J=2.1 \mathrm{~Hz} .2 \mathrm{H}) .5 .31$ (s, $2 \mathrm{H}), 5.80(\mathrm{~s} .2 \mathrm{H}) .7 .03-7.06(\mathrm{~m}, 2 \mathrm{H})$, $7.22-7.35(\mathrm{~m}, 1 \mathrm{lH}), 7.43-7.46(\mathrm{~m}, 2 \mathrm{H}) .7 .79(\mathrm{t}, J=2.1 \mathrm{~Hz}$, $1 \mathrm{H}) ;{ }^{12} \mathrm{C} \mathrm{NMR}\left(\mathrm{CDCl}_{3} .75 \mathrm{MHz}\right) \delta 46.93,50.04,58.61$, 122.43, 127.08, 127.78, 127.94, 128.02, 128.30, 128.79, 128.87. 129.44. 129.58, 133.64, 135.07. 137.82, 139.88, 161.39. 181.18.

Typical procedure for the synthesis of 4a. A mixture of $3 \mathbf{a}$ (153 mg, 0.4 mmol) and $\mathrm{PCC}\left(173 \mathrm{mg} .0 .8 \mathrm{mmol}\right.$ ) in $\mathrm{CH}_{2} \mathrm{Cl}_{2}$ $(5 \mathrm{~mL})$ was heated to reflux for $8 \mathrm{~h}$. The reaction mixture was filtered through a Celite pad and washed with $\mathrm{CH}_{2} \mathrm{Cl}_{2}$. The filtrates and washings were combined and solvent was removed. After column cluromatographic purification (hexanes/ ether, 1:1) conpound $t a$ was obtained as a white solid. 124 mg $(78 \%)$. Other compounds were șinthesized similarly and 
their spectroscopic data are as follows

Compound 4a: $78 \%$ : white solid mp $115-117^{\circ} \mathrm{C}$ : $\mathrm{IR}(\mathrm{KBr})$ 1716. $1673,1605,1448 \mathrm{~cm}^{-1}:{ }^{1} \mathrm{H} \mathrm{NMR}\left(\mathrm{CDCl}_{3}, 300 \mathrm{MHz}\right) \dot{\partial}$ 4.97 (s. 2H). 5.13 (s. 2H). 7.21-7.40 (m. 10H). 7.44-7.54 (m, $3 \mathrm{H}) .7 .65-7.69(\mathrm{~m} .2 \mathrm{H}) .7 .88(\mathrm{~s}, 1 \mathrm{H}):{ }^{13} \mathrm{C} \mathrm{NMR}\left(\mathrm{CDCl}_{3 .} 75\right.$ $\mathrm{MHz} \delta 44.71,53.06,113.33 .127 .75 .128 .03 .128 .25,128.37$, $128.76,129.11,129.15,129.22,132.89,134.42,136.24$. 137.41. 147.75, 150.89. 160.03, 190.68; ESMS $m / z 397$ $\left(\mathrm{M}^{-}+\mathrm{H}\right)$.

Compound +b: $73 \%$ : white solid mp 239-24I "C: IR (KBr) 1722. $1681,1659,1259 \mathrm{~cm}^{-1}:{ }^{1} \mathrm{H}$ NMR $\left(\mathrm{CDCl}_{3}, 300 \mathrm{MHz}\right)$ ò 7.26-7.31 (m. 2H). 7.36-7.57 (m. 1 l H). 7.80-7.84 (m. 2H). $8.16(\mathrm{~s}, 1 \mathrm{H}):{ }^{13} \mathrm{C} \mathrm{NMR}\left(\mathrm{CDCl}_{3} .75 \mathrm{MHz}\right)$ ò $114.30,126.34$. 128.20. 128.26. 128.98, 129.32. 129.40, 129.44. 129.69. 133.04. $13+.42,137.50,138 .+2,1+8.80,150.36,160.23,190.69$

Compound $+\mathrm{c}: 63 \%$; white solid, mp $192.193^{\circ} \mathrm{C}$; IR (KBr) $3188,169+1452 \mathrm{~cm}^{-1}:{ }^{1} \mathrm{H} \mathrm{NMR}\left(\mathrm{CDCl}_{3}, 300 \mathrm{MHz}\right)$ ò 5.00 (s. 2H). 7.30-7.43 (m. 7H). 7.52-7.58 (m. $1 \mathrm{H}) .7 .70-7.74(\mathrm{~m}$. 2H). 7.95 (s. IH). 8.77 (s. $1 \mathrm{H}):{ }^{13} \mathrm{C} \mathrm{NMR}\left(\mathrm{CDCl}_{3 .} .75 \mathrm{MHz}\right) \hat{o}$ 52.15. 114.09. 128.13. 128.37. 128.97, 129.31, 129.39, 133.16, $134.29 .137 .18,150.01,150.23,160.20 .190 .15$

Compound 4d: 80\%: white solid mp 240-241 "C: IR (KBr) 3163. 1709. $1305 \mathrm{~cm}^{-1}$ : ${ }^{1} \mathrm{H}$ NMR (CDCl. $\left.300 \mathrm{MHz}\right)$ ò 7.43-7.52(m. 7H). 7.60-7.65 (m. IH). 7.82-7.84 (m. 2H). $8.07(5,1 \mathrm{H}) .11 .80(\mathrm{~s}, 1 \mathrm{H}):{ }^{13} \mathrm{C}$ NMR $(\mathrm{CDCl} .75 \mathrm{MHz})$ ò 113.43. 127.01, 128.24. $128.71,129.15,129.31,132.93$. $137.64,138.45,149.60,149.95,161.32,190.52: \mathrm{ESIMS} m / z$ $293\left(\mathrm{M}^{+}+\mathrm{H}\right)$.

Compound 4e: 65\%: colorless oil: $\mathbb{R}$ (film) 1715. 1688. $1662,1449 \mathrm{~cm}^{-1}:{ }^{1} \mathrm{H} \mathrm{NMR}\left(\mathrm{CDCl}_{3}, 300 \mathrm{MHz}\right)$ ò 0.88 (t, $J=6.9$ $\mathrm{Hz}, 3 \mathrm{H}) .1 .26-1.35(\mathrm{~m} .4 \mathrm{H}) .1 .55-1.67(\mathrm{~m} .2 \mathrm{H}) .3 .03$ (t. $J=7.2$ Hz. $2 \mathrm{H}) .4 .98$ (s. 2H). $5.15(\mathrm{~s}, 2 \mathrm{H}), 7.23-7.40$ (m. $8 \mathrm{H})$. 7.44-7.48 (m, 2H), 8.2l (s, lH); ${ }^{j 3} \mathrm{C} \mathrm{NMR}\left(\mathrm{CDCl}_{3}, 75 \mathrm{MHz}\right)$ ó 13.90, 22.48, 23.40, 31.35, 42.58, 44.81, 53.35, 112.16. $127.76,128.22,128.45,128.80,128.89,129.17,134.44$. 136.30, 148.33, 150.97, 160.55, 197.06: ESIMS $m / z \quad 391$ $\left(\mathrm{M}^{-}+\mathrm{H}\right)$.

Compound 4f: $66 \%$ : white solid. mp $135-137^{\circ} \mathrm{C}$ : IR (KBr) 1692. 1666, 1224 $\mathrm{cm}^{-1}:{ }^{\mathrm{l}} \mathrm{H}$ NMR (CDCl $\left.3.300 \mathrm{MHz}\right)$ ò 5.56 (s. $2 \mathrm{H}) .5 .76(\mathrm{~s}, 2 \mathrm{H}) .7 .22-7.56(\mathrm{~m} .13 \mathrm{H}), 7.66-7.69(\mathrm{~m}, 2 \mathrm{H})$. 7.95 (s. $1 \mathrm{H}):{ }^{13} \mathrm{C} \mathrm{NMR}\left(\mathrm{CDCl}_{3} .75 \mathrm{MHz}\right)$ oे 50.77, 59.26. 115.81. 127.58. 128.12. 128.18, 128.24, 128.59, 128.81 . $129.19,129.29,133.24,134.03,135.49,136.96,146.71$. 157.79. 177.96, 190.31: ESIMS $m / z+13(\mathrm{M}+\mathrm{H})$.

Acknowledgments. This work was supported by the Korea Research Foundation Grant funded by the Korean Governument
(MOEHRD. KRF-2007-313-C00417). Spectroscopic data was obtained from the Korea Basic Science Institute. Gwangju branch

\section{References and Notes}

1. Modifications of 5-and 6-positions of uracil derivatives, see: (a) Abarbri, M: Knochel, P. Syntett 1999, 1577-1578. (b) Singh, P: Paul, K. Holzer, W. Ong. Biomol Chem 2005, 3.3958-3965. (c) Berillon, L: Wagner, R.; Knochel, P. J. Org. Chem. 1998, 63, 9117-9121. (d) Kumar, S.; Malik, V; Kaur, N.; Kaur, K. Tetrahedron Lett. 2006, $47,8483-8487$

2. For the synthesis and applications of 5-arovluracil derivatives, see: (a) Abarbri, M.; Thibonnet, T.; Berillon, L.; Dehmel, F.; Rottlander, M.: Knochel, P. J. Org. Chem 2000, 65, 4618-4634. (b) Matsumoto, N:- Takahashi, M. Tetruhedron 2002, 58 , 10073-10079. (c) Singh. P.; Pall, K. Bioong. Med. Chem. 2006, 14.7183-7186. (d) Singh, P.: Paul. K. Bioong. Med. Chem. 2006. 14,8622-8625. (e) Kim, J. N.; Ryu, E. K. J. Org. Chent 1992, 57, 1088-1092. (f) Bernier, T.-L.: Henichart, J.-P.: Warin, V.; Trentesaux, C.. Jardillier, J.C. J. Ong. Chem. 1985, 28,497-502. (g) Jachak, M. N.: Tantak, C. D.; Toche, R. B.; Badgujar, N. S. Monatsh Chem. 2004. 135, 1529-1538.

3. For the synthesis of pyrimidine and related compounds from Bav lis-Hillman adducts, see: (a) Nag, S.; Yadav, G. P.; Maulik, P. R.: Batra, S. Symthesis 2007, $911-917$ (b) Kim, .T. M.: Kim, S. H.: Kim, I. N. Bull. Konean Chem. Soc, 2007, 28, 2505-2507. (c) Basavaiah, D.; Satyanarayana, T. Tetrohedron Leth. 2002, t3, 4301-4303. (d) Pathak, R.: Roy, A. K.: Batra, S. Synlett 2005, $848-850$.

4. For general review on Baylis-Hillman reaction, see: (a) Basalvaiah. D.: Rao. A. I.: Satyanarayana. T. Chem. Rev. 2003. 103, 81 1-891. (b) Ciganek, E. In Organic Reactions; Paquette, L. A., Ed: Tohn Wiley \& Sons: New York, 1997; Vol. 51, pp 201-350. (c) Basavaiah, D.; Rao, P. D.: Hyma, R. S. Tetrahedron 1996, 52,8001-8062. (d) Kim, J. N.: Lee, K. Y. Cum: Org. Chem. 2002, 6, 627-645. (e) Lee, K. Y:; Gowrisankar, S.; Kim, J. N. Bull. Kowean Chem. Soc. 2005, 26, 1481-1490. (f) Singh, V:; Batra, S. Tetrahtedron 2008, 64, $4511-4574$ and further references cited therein

5. Lee, C. G.: Gowrisarkar, S.; Kim, J. N. Bull. Korean Chem. Soc. $2005,26,481-484$

6. The oxidation of 2 a with $\mathrm{PCC}\left(4.0\right.$ equiv) $/ \mathrm{CH}_{2} \mathrm{Cl}_{2}$ (reflux, $72 \mathrm{~h}$ ) afforded $19 \%$ of product $4 a_{\text {, and }} 2 \mathrm{a}$ was recovered in $70 \%$. The reaction of $\mathrm{CrO}_{3}(5.0$ equiv)/AcOH (reflux, $2 \mathrm{~h}$ ) produced ta in $27 \%$. Oxidation of 2 a with $\mathrm{SeO}_{2}(2.0$ equiv) EtOH (reflux, $2+\mathrm{h}$ ) or $\mathrm{KMnO}_{4}\left(2.0\right.$ equiv)/aqueous $\mathrm{ClCH}_{2} \mathrm{CH}_{2} \mathrm{Cl}$ (reflux, $24 \mathrm{~h}$ ) was ineffective.

7. For the PCC oxidation of similar compounds, (a) Kim, S. I.: Lee, H. S.; Kim, I. N. Tetrahedron Lett. 2007, 18, 1069-1072. (b) Kim, S. C.; Lee, H. S.: Kim, T. N. Bull. Korean Chem. Soc. 2007, 28, 147-150. (c) Kim. S. H.; Kim. S. H.: Kim. J. N. Bull. Konean Chem. Soc. 2008, 29, 2039-2042. 